OMAE2014-24587

\title{
THE EFFECT OF THE COMPUTATIONAL GRID SIZE ON THE PREDICTION OF A FLAMMABLE CLOUD DISPERSION
}

\author{
Adriana Miralles Schleder \\ Naval Architecture and Ocean Engineering Department \\ University of São Paulo \\ Sao Paulo - Brazil \\ adrianamiralles@usp.br \\ Elsa Pastor Ferrer \\ Department of Chemical Engineering \\ Centre for Technological Risk Studies \\ Universitat Politècnica de Catalunya - BarcelonaTech \\ Barcelona - Spain \\ elsa.pastor@upc.edu
}

\author{
Marcelo Ramos Martins \\ Naval Architecture and Ocean Engineering Department \\ University of São Paulo \\ Sao Paulo - Brazil \\ mrmartin@usp.br \\ Eulàlia Planas Cuchi \\ Department of Chemical Engineering \\ Centre for Technological Risk Studies \\ Universitat Politècnica de Catalunya - BarcelonaTech \\ Barcelona - Spain \\ eulalia.planas@upc.edu
}

\section{ABSTRACT}

The consequence analysis is used to define the extent and nature of effects caused by undesired events being of great help when quantifying the damage caused by such events. For the case of leaking of flammable and/or toxic materials, effects are analyzed for explosions, fires and toxicity. Specific models are used to analyze the spills or jets of gas or liquids, gas dispersions, explosions and fires. The central step in the analysis of consequences in such cases is to determine the concentration of the vapor cloud of hazardous substances released into the atmosphere, in space and time. With the computational advances, CFD tools are being used to simulate short and medium scale gas dispersion events, especially in scenarios where there is a complex geometry. However, the accuracy of the simulation strongly depends on diverse simulation parameters, being of particular importance the grid resolution. This study investigates the effects of the computational grid size on the prediction of a cloud dispersion considering both the accuracy and the computational cost.

Experimental data is compared with the predicted values obtained by means of CFD simulation, exploring and discussing the influence of the grid size on cloud concentration the predicted values.

This study contributes to optimize CFD simulation settings concerning grid definition when applied to analyses of consequences in environments with complex geometry.

\section{INTRODUCTION}

As a result of the industrial and technological development, the presence of flammable and toxic substances has significantly increased in a number of activities. While flammable substances are used as energy sources, toxic substances are used in a huge number of industrial processes, and frequently the flammable and toxic substances are present in the same process. Activities related to the supply chain of oil and its derivatives is a current example; these substances are present in the activities of offshore and onshore production plants, in the storage and transport process and in the process of delivery to the final consumer.

Although these substances are essential nowadays, there are risks involved in their manipulation, storage and transportation that should be controlled whenever possible. The consequence analysis is used to define the extent and nature of effects caused by undesired events on individuals, buildings, equipment and on the environment. For the case of leaking flammable and/or toxic materials, consequences are analyzed for explosions, fires and toxicity.

The central step in this type of analysis is to determine the concentration of the vapor cloud of hazardous substances released into the atmosphere, in space and time. On the basis of this approach, the use of numerical methods associated with different algorithms of computational fluid dynamics (CFD) to 
perform consequences analysis has grown in recent years [1-4]. CFD is found in some commercial software tools such as ANSYS, FLACS, FLUENT, PHOENIX and COMSOL Multiphysics. The CFD tools transform the governing equations of the fundamental physical principles of fluid flow in discretized algebraic forms, which are solved to find the flow field values in time and space [5].

Although CFD tools have proven promising to perform analyzes of consequences in environments with complex geometry, there are still challenges to be overcome, as shown by Plasmans et al.[6]; previous studies have shown that large differences may arise between the results when working with different tools and/or different CFD analysts to assess the same scenario. The simulation results can be very sensitive to the wide range of computational parameters that must be set by the user; for a typical simulation, the user needs to select the variables of interest, turbulence models, computational domain, computational mesh, boundary conditions, methods of discretization and convergence criteria among others. In this context, the study presented here intends to investigate the effects of the computational grid size on the prediction of a cloud dispersion simulation. This study is part of a research project that aims to provide key information for decision making about the use of CFD tools on cloud dispersion simulation for different scenarios of interest, such as those containing barriers to dispersion, and therefore contributing to optimize the accuracy of the results.

In order to perform this analysis, experimental data are compared with the simulated CFD values and the influence of the grid size of the simulated scenario on the predicted values of the cloud features is explored and discussed. Two scenarios are presented: a scenario of a jet release of dense gas in open and flat terrain and a similar release with the presence of a fence. Next, a grid dependence analysis is performed in order to verify the effects of the grid on the prediction of the cloud dispersion.

\section{COMPUTATIONAL FLUID DYNAMICS}

As previously mentioned, the CFD tools compute the flow field values by the equations of the fundamental physical principles of a fluid flow. The physical aspects of any fluid flow are governed by three principles: mass is conserved, Newton's second law (momentum equation) is fulfilled and energy is conserved. These principles are expressed in integral equations or partial differential equations being the most common form the Navier-Stokes equations for viscous flows and the Euler equations for inviscid flows.

The commercial tool FLACS was used to perform this study [7]; FLACS incorporates a three-dimensional model for the simulation of vapor cloud dispersion and a water-based model for the simulation of pool spreading and vaporization. The pool vaporization is evaluated based on heat transfer from the substrate, convective heat transfer from the air, solar radiation, turbulence levels, local wind speed and local vapor pressure. All these phenomena are calculate at each time step and locally, for each grid cell [8]. The cloud concentration will be also influenced by atmospheric turbulence, atmospheric stability and density changes.

As presented by Gavelli, Scott and Hansen [8], the model available in FLACS solves Reynolds Averaged Navier-Stokes (RANS) equations based on the standard " $k-\varepsilon$ " model of Launder and Spalding [9]. According to HSE [9], RANS approach is widely accepted and documented; it is based on the concept of separating the fluid velocity components and scalar quantities (such as pressure, temperature, concentration) into mean and fluctuating components, then transport equations are used to evaluate the model. The standard " $k-\varepsilon$ " model of Launder and Spalding [9] describes the turbulence in function of the magnitudes of two turbulence quantities: the turbulence kinetic energy $(\mathrm{k})$ and its dissipation rate $(\varepsilon)$; they are calculated from transport equations solved at the same time with those governing the mean flow behavior.

\section{GRID}

In order to solve the physics of the flow field it is necessary to divide the flow domain in small subdomains, which implies the generation of a grid (or mesh) of cells also defined as control volumes. The geometry and size of these cells coupled with the numerical method used to solve the governing equations are determining aspects when evaluating the accuracy and the resolution time of a simulation. As presented by Thompson, et al. [11], the grid cells must be sufficiently small to provide an accurate numerical approximation, but they cannot be so small that the solution is impractical to obtain. Thus, usually the mesh is refined in the regions of interest as around the main obstacles affecting the cloud dispersion and nearby the source terms (micro grid) and is smoothly increased to the prevailing grid (macro grid).

This mesh can be structured meaning that the lines are based on coordinate directions or unstructured i.e. with no relation with coordinate directions; in the first case the mesh consists of quadrilateral cells in 2D, or hexahedral cells in $3 \mathrm{D}$, and the unstructured mesh usually consists of triangles in 2D and tetrahedral in 3D, but cells can be of any form. Structured grids usually imply shorter time resolution, however the unstructured meshes may better represent the geometry and have been gaining popularity in recent years; for example Yasushi[12] presents a discussion about the development of efficient computational analysis using unstructured grid and Luo \& Spiegel [13] propose a method to generate a hybrid mesh (coupling strutucred and unstructured grid). The basic concepts of grid generation are found in [5] and a detailed discussion about the influence of grid in CFD applications can be found in Thompson, et al. [11].

As presented by Gavelli, Scott and Hansen [8], FLACS solves the conservation equations for mass, mass fraction of species, energy and momentum using a finite volume method on a 3-D Cartesian grid, where complex geometries are represented by a porosity concept.

The mesh implemented in FLACS is composed of cubic or cuboid-shape cells defined by grid lines that are horizontal and vertical lines related with coordinate directions; the mesh 
resolution can be adjusted in any Cartesian direction, however, it is not possible to build the mesh with inclined or curved lines [7].

As presented by Arntzen [13], it is important that all objects are well geometrically represented on the grid when evaluating the effects of the obstacles. Obstacles such as pipes are represented in FLACS defining an area porosity on the control volume faces and a volume porosity referred to the interior of the control volume; the porosity is the fraction of the area/volume that is accessible for a fluid to flow. There are three porosities areas for each control volume; one for each of the surfaces on each direction of the control volume such that the accessible area for the fluid can be represented in all directions. The porosity is represented by a value between 0 and 1 , where 0 means that the control volume is completely blocked and 1 means that the control volume is completely unblocked.

The porosity of the cell face will be calculated by the smallest porosity in any plane between cell centers. Figure 1, adapted from Arntzen [13], shows an example: two cells of the grid containing blocks and cylinders smaller than the grid cell. The porosity in face $e$ is actually $100 \%$ since none of the objects matches this face; however, to take into account the effects of the small objects, the porosity in this face will be $50 \%$ : the smallest porosity in any plane located between $P$ and $W$ (the centerlines of the grid cells).

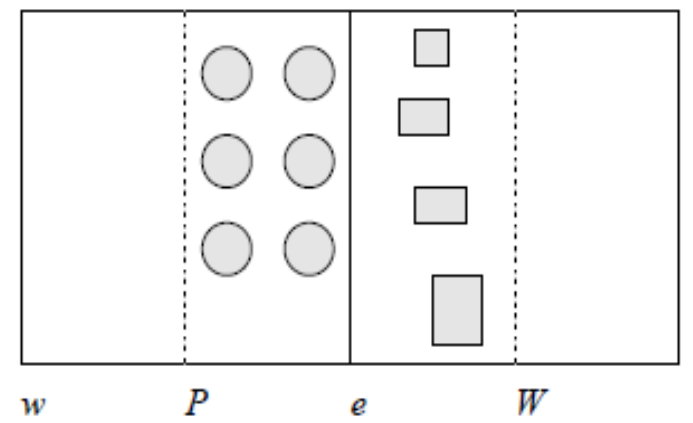

Figure 1 - Two cells containing sub-grid geometry Adapted from Arntzen [13]

The grid guidelines of FLACS recommend that the large objects (objects larger than 1.5 cell) should be aligned with the grid lines, since the program that evaluates the porosities adjusts automatically the large objects to match with the mesh. This can cause some undesired situations, like leak corners (i.e. if a wall is moved to match the closest grid line, a space between this wall and the nearest object may appear allowing the passage of the fluid where originally it would not be possible). For sloping cases a "staircase" representation is used [7].

The objects will be adjusted to match the grid lines; however, in many cases, it is not possible to represent suitably small objects in the grid, and thus subgrid models must treat these objects.

Subgrid objects (objects that are smaller than a grid cell) contribute to turbulence generation; in case of small objects, the flow kinetic energy lost due to drag forces is compensated as a source term for turbulent energy. In FLACS, this contribution is calculated for objects smaller than two control volumes; the turbulence contribution increases when the object dimensions decrease such that there is a gradual transition from the subgrid to macrogrid representation.

Finally, the grid guidelines of FLACS also recommend a three-step procedure for dispersion analysis: to cover the computational domain with a uniform grid, to refine the grid in the region of the release and to stretch the grid outside the main region towards the boundaries [7]. Additionally, the guidelines suggest that initially the grid be represented by $1-1.5 \mathrm{~m}$ edge cubes for offshore modules higher than $8.5 \mathrm{~m}$ and equal to 0.5 $\mathrm{m}$ for lower modules and for terrains with slope the grid must be refined (in a range between 0.1 and $0.5 \mathrm{~m}$ ) in vertical direction.

\section{BASELINE SCENARIO}

In order to perform the grid analysis it is necessary to choose a baseline scenario from which make the grid alterations to observe potential changes in simulation results. Two trials of the field tests performed by Health and Safety Laboratory (HSL) at the HSL laboratories in Buxton, England [15] were chosen as baseline scenarios.

In the HSL trials, liquefied propane was released at rates up to $4.9 \mathrm{~kg} / \mathrm{s}$, at a height of $1.5 \mathrm{~m}$. The resulting vapor cloud was characterized to determine the cloud temperature and concentration of propane vapor at different distances from the release point. The trials set-up comprised a liquefied propane storage facility, a release system and a discharge area in which were produced the vapor clouds. The layout of the trials site is shown in Figure 2, at the top the plant of the trial site and at the bottom the representation of the sensors height.

The discharge length is aligned with the prevailing wind, having its long dimension running south-west to northeast. Open fields are adjacent to the north and west of the area, and a deep valley forms the southern and eastern perimeter. Sensors were placed over a $600 \mathrm{~m}^{2}$ area $(100 \mathrm{~m}$ in downwind direction and $6 \mathrm{~m}$ in crosswind direction), located within the gas dispersion site; they were located at heights of $0.20,0.85$ or $1.50 \mathrm{~m}$ above the ground on the first $40 \mathrm{~m}$ of the centerline of the site and at a height of $0.20 \mathrm{~m}$ in all the other points, as indicated at the bottom of Figure 2.

Some of the trials undertaken were designed to investigate the influence of an obstruction placed in the path of the vapor flow. From preliminary observations of the gas flow, a $1 \mathrm{~m}$ high fence was chosen to be a suitable obstruction. Using this height, the top of the fence was approximately in the middle of the gas cloud height, allowing a significant volume of gas to flow unobstructed, whilst at the same time providing an obstruction for the lower part of the cloud. The fence was constructed using $2 \mathrm{~m}$ by $1 \mathrm{~m}$ steel sheets; ten sheets were used, producing a $20 \mathrm{~m}$ long fence, which was positioned $15 \mathrm{~m}$ from the release nozzle, perpendicular to the centerline of the trials site. The fence was centered so that there was $10 \mathrm{~m}$ of fence at either side of the centerline. 


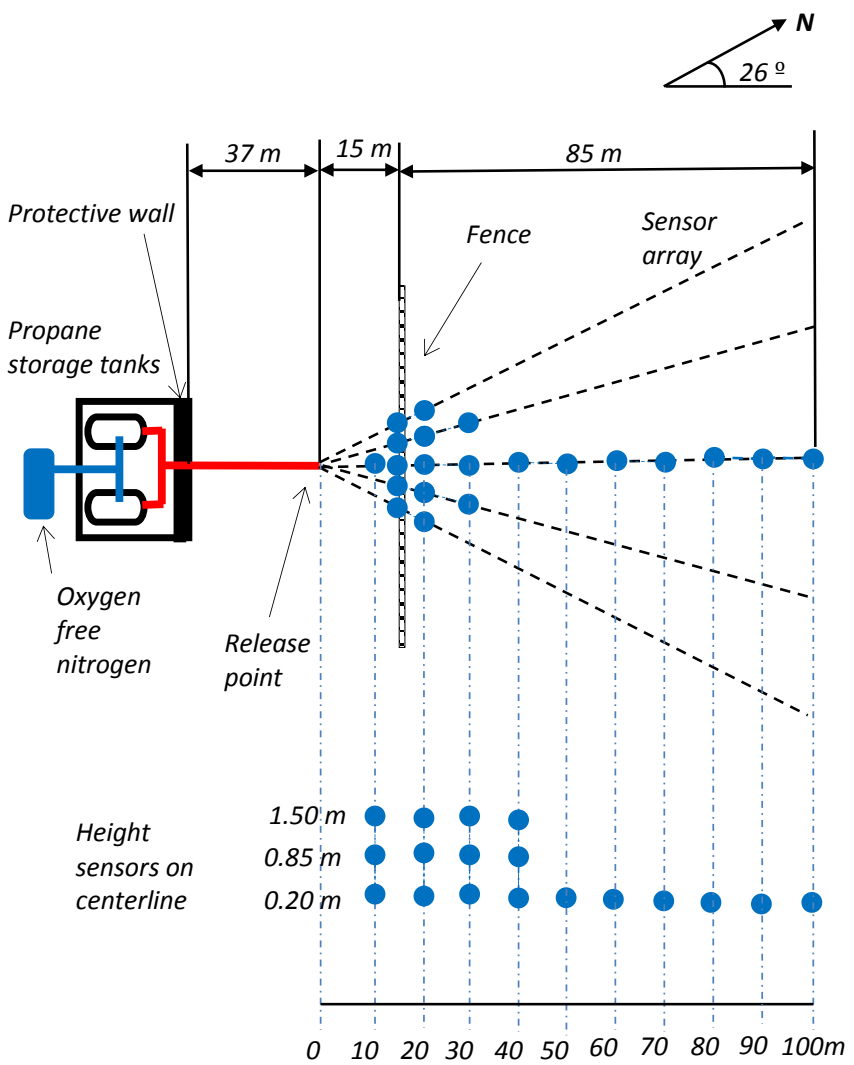

Figure 2 - Test layout. Adapted from Butler \& Royle [15]

Trials 8 and 11 of this field tests were selected as baseline scenarios (B1 and B2) to perform the grid dependence analysis. These trials were chosen due to the similarity of initial conditions. The former test presents an unobstructed scenario while the other presents the scenario with a fence ac ting as an obstruction to cloud dispersion. The input parameters used to perform the simulations are presented in Table 1 .

Table 1 - Scenario conditions of baseline scenarios

\begin{tabular}{lccc}
\hline Variable & Unit & B1 & B2 \\
\hline Ambient Temperature & ${ }^{\circ} \mathrm{C}$ & 14.5 & 17.5 \\
\hline Atmospheric pressure & $\mathrm{bar}$ & 1 & 1 \\
\hline Wind speed & $\mathrm{m} / \mathrm{s}$ & 3.0 & 5.0 \\
\hline Pasquill Class & - & $\mathrm{D}$ & $\mathrm{D}$ \\
\hline Wind direction & $\mathrm{o}$ & $195-225$ & $110-225$ \\
\hline Relativity humidity & $\%$ & 63 & 63 \\
\hline Ground roughness & $\mathrm{m}$ & 0.03 & 0.03 \\
\hline Temperature release & $\mathrm{o} C$ & 11.96 & 11.26 \\
\hline Pressure release & $\mathrm{bar}$ & 7.87 & 7.58 \\
\hline Discharge rate & $\mathrm{kg} / \mathrm{s}$ & $2.5 \pm 0.3$ & $3.4 \pm 0.3$ \\
\hline Discharge direction & - & horizontal & horizontal \\
\hline Release duration & $\mathrm{s}$ & 131 & 141 \\
\hline Discharge height & $\mathrm{m}$ & 1.5 & 1.5 \\
\hline
\end{tabular}

The domain was divided in three areas: the first one around the release point (micro grid), formed by the cells where the leak takes place and the adjacent cells (the regions near the height of $1.5 \mathrm{~m}$ and near the point $(0,0)$ in $\mathrm{X}$ and $\mathrm{Y}$ directions); the second, the prevailing grid formed by the area where the dispersion is expected (macro grid); and the third, the stretched area in the far field where no relevant concentrations are expected. The transitions among these areas are made gradually in order to obtain stable simulations; the cells are increases gradually from one region to another of the grid such as the maximum ratio between one cell and the next one is two.

The domain was discretized using a single block Cartesian grid; the domain and the grid of the baseline scenarios were built following the guidelines of the FLACS user manual [7]. An orthogonal base $\mathrm{X}, \mathrm{Y}$ and $\mathrm{Z}$ was used, being; the $\mathrm{X}$ direction horizontal and parallel to wind, the $\mathrm{Y}$ direction perpendicular to the wind and horizontal and the $\mathrm{Z}$ direction vertical, being the point $(0,0,1.5)$ coinciding with the release point. The computational domain extended $170 \mathrm{~m}$ in the $\mathrm{X}$ direction (from $20 \mathrm{~m}$ upwind to $150 \mathrm{~m}$ downwind from the release point), $30 \mathrm{~m}$ in the $\mathrm{Y}$ direction (symmetric crosswind plan from the release point) and $10 \mathrm{~m}$ in the $\mathrm{Z}$ direction; the cells were initially represented by $1 \mathrm{~m}$ edge cubes (forming the macro grid).

Concerning the micro grid dimensioning, the guidelines [7] specify that the area of the expanded jet must be solved in only one cell and that the area of this cell across the jet should be larger than the area of the expanded jet but not larger than twice. Therefore, the jet area expected after the expansion at ambient pressure was estimated and the dimensions of the face cell across the jet defined so that the area fell between these limits.

Additionally it is recommended that the aspect ratio (the ratio between the smallest and largest side of the cell) of the refined leak cells is not larger than five due to stability of the numerical solution. Once the dimensions of the cells around the leak were defined, cells nearby were smoothly increased to the macro grid resolution.

Thus, in B1 scenario, the width and height of the micro grid cells were fixed at $0.15 \mathrm{~m}$ (as a function of the jet area expected after the expansion at ambient pressure) and, in order to maintain the aspect ratio smaller than 5 , the length of the cells was fixed at $0.5 \mathrm{~m}$. In B2 scenario, the width and height of the micro grid cells were fixed at $0.17 \mathrm{~m}$ and the length of the cells was fixed at $0.86 \mathrm{~m}$.

Lastly, in both scenarios, the grid was stretched in $\mathrm{X}$ direction away from the leakage point (the length of cell grows continuously at a rate of 1.15 to provide a smooth growth with increasing distance from the source): the cells are stretched after $100 \mathrm{~m}$ from the leakage point because after this distance are not expected significant concentrations of gas. Thus, the micro grid is defined in function of the jet as previously mentioned, the stretched grid is defined in the far field (after $100 \mathrm{~m}$ from the leakage point) by cells larger than the macro grid cells and the macro grid is defined by the initial grid of 1 m edge cubes. 
Taking into account that the focus of this study is the dispersion of a cloud the main variable of interest was defined as the concentration of this cloud. Monitoring points were inserted in the simulation specifications at the same points where the gas sensors were placed in the field tests which allowed the measured values of concentration to be compared with the simulated values.

\section{GRID DEPENDENCE SIMULATIONS}

The grid dependence analysis was performed in three phases: first, the influence of variations of up to $20 \%$ in the dimensions of the macro grid was studied: next, it followed the analysis of the variations of up to $20 \%$ in the dimensions of the micro grid; and finally, the effects of variations by more than $20 \%$ in the macro grid were examined.

In order to verify the grid dependence, each dimension of the macro grid cells was changed independently of the others; for example, when the width was increased by $10 \%$, the other dimensions remained the same as those defined in the baseline scenario. Each dimension was increased and decreased by $10 \%$ and $20 \%$.

The same approach was used for both baselines scenarios. The micro grid around the release point was not modified when doing this analysis. Table 2 shows the simulations executed for each scenario, in which each dimension of the macro grids cells is varied.

Table 2 - Simulations to verify grid dependence

\begin{tabular}{|c|c|c|c|}
\hline \multirow{2}{*}{ Scenario } & \multicolumn{3}{|c|}{ Dimensions of the macro grid cells } \\
\hline & Length $[\mathrm{m}]$ & With $[\mathrm{m}]$ & Height $[\mathrm{m}]$ \\
\hline B & 1 & 1 & 1 \\
\hline L1 & 1.2 & 1 & 1 \\
\hline L2 & 1.1 & 1 & 1 \\
\hline L3 & 0.9 & 1 & 1 \\
\hline $\mathrm{L} 4$ & 0.8 & 1 & 1 \\
\hline W1 & 1 & 1.2 & 1 \\
\hline W2 & 1 & 1.1 & 1 \\
\hline W3 & 1 & 0.9 & 1 \\
\hline W4 & 1 & 0.8 & 1 \\
\hline $\mathrm{H} 1$ & 1 & 1 & 1.2 \\
\hline $\mathrm{H} 2$ & 1 & 1 & 1.1 \\
\hline H3 & 1 & 1 & 0.9 \\
\hline $\mathrm{H} 4$ & 1 & 1 & 0.8 \\
\hline
\end{tabular}

The simulated values after the variation on each grid dimension of baseline scenarios B1 and B2 were compared with the experimental data. Only the results obtained after the variation of the cell height in scenario B2 are presented here (Figure 3). In this figure, the blue line "Exp" represents the experimental data, the line B2 represents the predicted values obtained using the initial grid for baseline scenario B2 described in the previous section, the lines $\mathrm{H} 1$ and $\mathrm{H} 2$ represent the predicted values obtained using the cell height increased $20 \%$ and $10 \%$ respectively; and the lines $\mathrm{H} 3$ and $\mathrm{H} 4$ represent the decrease by $10 \%$ and $20 \%$ respectively (according to the Table 2). It is worth noting that with the refinement of height the results improve and approach to the experimental values. It is also possible to see significant effects concentrated in the region near field and minor effects in far field. After $15 \mathrm{~m}$ from the release point there is a significantly decrease on the concentration because the presence of the fence that obstructs the cloud dispersion.

The HSE in the Model Evaluation Protocol (MEP) recommends the use of a factor of 2 range to validation purposes of CFD models [16]. Although this paper does not intend to perform a validation exercise, the results were compared with this recommended range. For the initial grid, $50 \%$ of the simulated values fitted well to this range; the major discrepancies found are related to very low values of concentration.

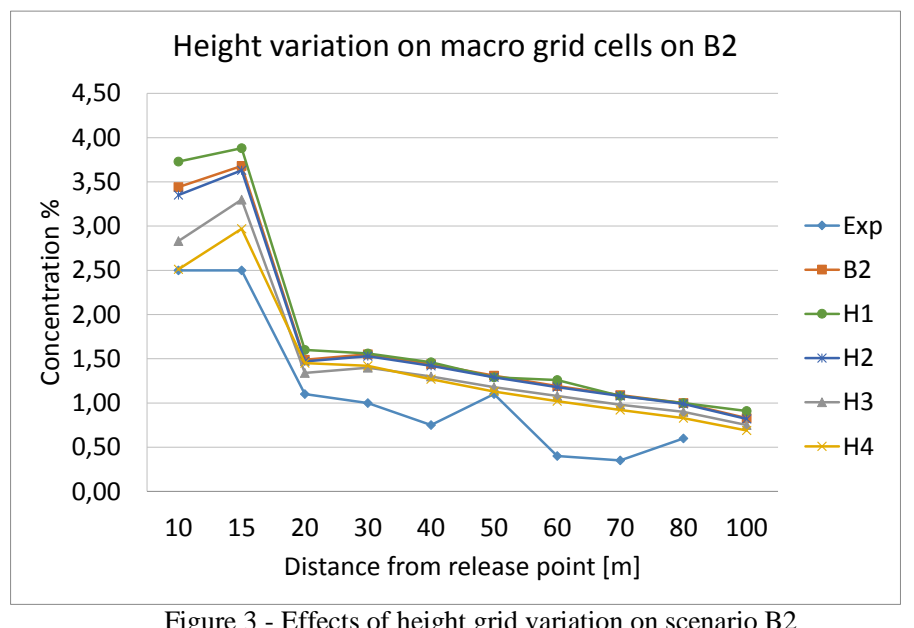

In both scenarios, B1 and B2, the change that caused the minor influence was the alteration of the control volume width ( $\mathrm{Y}$ direction), in which the major relative variation with respect to the baseline scenario B1 was about $2 \%$. This is the control volume side across to the wind direction and to leak direction; thus this minor influence is expected since the flow is less affected in this direction by the turbulence forces of the source term and by the wind.

Figure 4 and Figure 5 show the comparison among the best results obtained with variations in each dimension of scenarios $\mathrm{B} 1$ and $\mathrm{B} 2$ respectively; it is possible to see that the largest variation was achieved with the variation of height (lines H4, being these lines more distant from the baseline scenario tendency than the others), in this case the relative variation on results reached $27 \%$ (with respect to the baseline scenario B2). The closest results to the experimental data were obtained by the alteration of height; this occurs because the substance is a dense gas. The parcel related to weight in the momentum governing equation (Newton's second law) has a significant impact in the results and therefore the refinement in the control volume height allows a better representation of this parcel. Moreover, the better representation of this parcel allows a better representation of the fence effects on scenario B2 (Figure 5); with a more refined grid the cloud simulated is more similar 
to the experimental cloud which is suffering the influence of the turbulence generated by the fence. Finally, in both scenarios, it is possible to see significant effects concentrated in the region near field and minor effects in far field. This occurs due to the turbulence effects of the source term on the flow, since in the initial phase of the dispersion the features of the source term define the flow.

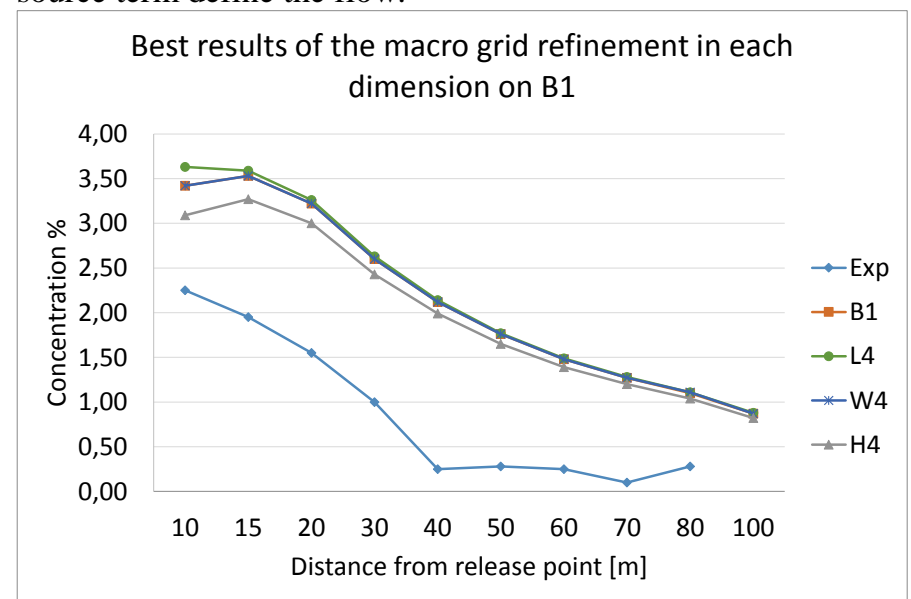

Figure 4 - Comparison among variations in each dimension of the macro grid cells on $\mathrm{B} 1$

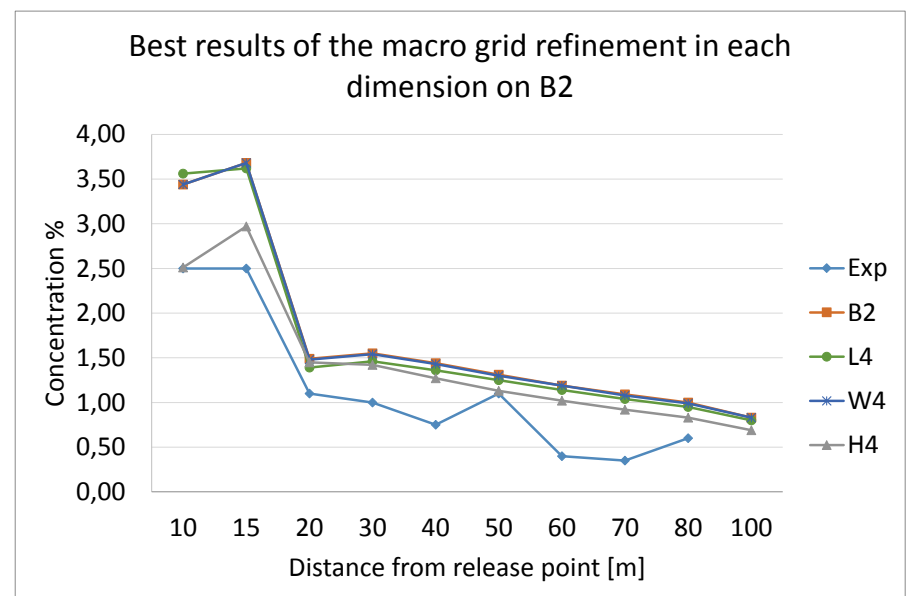

Figure 5 - Comparison among variations in each dimension of the macro grid cells on $\mathrm{B} 2$

Regarding the runtime of the simulations, the refinement of one dimension of grid by a rate of $20 \%$ resulted in an increase of approximately 2 hours of runtime; for scenario B1, it increased from 8.4 to 10.3 hours and for scenario B2 from 9.5 to 11.6 hours (Simulations performed using randomly eight cores Intel Xeon Quad-Core 5520 de $2.26 \mathrm{GHz}$ ).

Next, a dependence grid analysis in the micro grid around the release point was performed in order to obtain more information about the influence of the grid in the first region of the flow. As performed in the macro grid analysis, each dimension of the control volumes in the discharge region was changed independently of the others; each one was increased and decreased by $20 \%$. The same approach was used to both baselines scenarios. The macro grid around the release point was not modified in this analysis. Table 3 shows the simulations executed for each baseline scenario.

Table 3 - Simulations to verify micro grid dependence

\begin{tabular}{ccccc}
\hline Scenario & $\begin{array}{c}\text { Dimensions of the cells in the } \\
\text { area of the expanded jet } \\
\text { Length } \\
{[\mathrm{m}]}\end{array}$ & With $[\mathrm{m}]$ & $\begin{array}{c}\text { Height } \\
{[\mathrm{m}]}\end{array}$ & Simulations \\
\hline B1 & $\mathbf{0 . 5}$ & $\mathbf{0 . 1 5}$ & $\mathbf{0 . 1 5}$ & 1 \\
\hline L5 & 0.6 & 0.15 & 0.15 & 1 \\
\hline L6 & 0.4 & 0.15 & 0.15 & 1 \\
\hline W5 & 0.5 & 0.18 & 0.15 & 1 \\
\hline W6 & 0.5 & 0.12 & 0.15 & 1 \\
\hline H5 & 0.5 & 0.15 & 0.18 & 1 \\
\hline H6 & 0.5 & 0.15 & 0.12 & 1 \\
\hline B2 & $\mathbf{0 . 8 6}$ & $\mathbf{0 . 1 7}$ & $\mathbf{0 . 1 7}$ & 1 \\
\hline L5 & 1.03 & 0.17 & 0.17 & 1 \\
\hline L6 & 0.69 & 0.17 & 0.17 & 1 \\
\hline W5 & 0.86 & 0.20 & 0.17 & 1 \\
\hline W6 & 0.86 & 0.14 & 0.17 & 1 \\
\hline H5 & 0.86 & 0.17 & 0.20 & 1 \\
\hline H6 & 0.86 & 0.17 & 0.14 & 1 \\
\hline-95 & & & \\
\hline
\end{tabular}

As observed in the macro grid analysis, the change that caused minor influences was the alteration of the control volume width. The major effects were again concentrated in the region near the release point and near the obstacles and decreased in the far field.

Additionally, comparing the results among the variations in the three dimensions of the control volume, it could be seen that the closest results to the experimental data were obtained again by altering the height. Figure 6 and Figure 7 present the comparisons among results for B1 and B2 baseline scenarios, and it can be clearly observed how the best results are relative to lines H6. The major relative variation with respect to the baseline scenario (B2) was about $28 \%$. As in the previous analysis, the parcel of the weight in the momentum governing equation has a significant impact in the results.

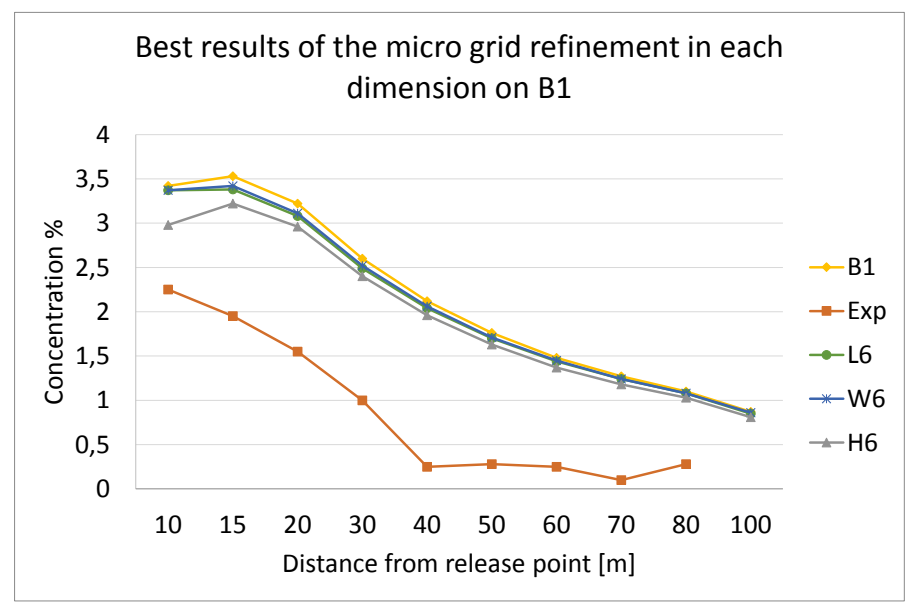

Figure 6-Comparison among variations in each dimension of the micro grid cells on B1 


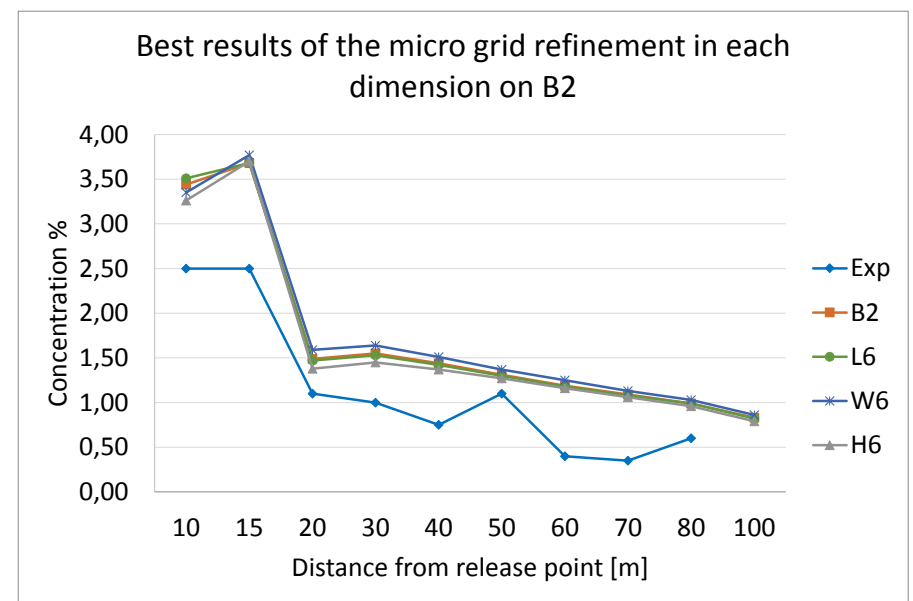

Figure 7 - Comparison among variations in each dimension of the micro grid cells on $\mathrm{B} 2$

Comparing the results of the micro and macro grid refinement, it can be noted that the micro grid refinement produces roughly the same improvement on simulating scenario B1 of those achieved by the macro grid refinement. Concerning scenario B2, the refinement of the macro grid contributes more to the accuracy of the results since the source term and the effects of the turbulence generated by the fence are better represented, while the refinement in micro grid only improves the representation of the source term.

Concerning to the simulation runtime, as in the macro grid analysis, the refinement on micro grid by a rate of $20 \%$ resulted in an increase of approximately 2 hours of runtime and when the micro grid was stretched by a rate of $20 \%$ the runtime decrease also approximately 2 hours.

After observing that the height refinement of the macro grid produced better simulation results, especially in the scenario with a barrier that is the focus of this study, shorter grids were tested; the height of the cells of the baseline scenarios were decreased also by $30 \%, 40 \%, 50 \%$ and $60 \%$.

The results are presented in figures below (Figure 8 and Figure 9 for scenarios B1 and B2 respectively); there is an improvement on the results with the grid refinement until the rate of $50 \%$ (lines $\mathrm{H} 3-10 \%, \mathrm{H} 4-20 \%, \mathrm{H} 7-30 \%, \mathrm{H} 8-40 \%$ and $\mathrm{H} 9-50 \%$ respectively); next, doing the decrease of $60 \%$ in the height of the cells (line H10), the distance between the numerical results and the experimental data increases. Comparing the results of the original grid with the grid refined in $50 \%$ (line $\mathrm{H} 9$ of the Figure 9) results improved $12 \%$. This occurs because the aspect ratio between the cells dimensions increases with the reduction of cells' height and the maintenance of the other dimensions; until a ratio of 2 the results are improved, however for ratios larger than 2, the results become as inaccurate as with the original grid (non refined grid).

Additionally it is worth to note that after the improvement reached by the grid refinement, the percentage of simulated values that fit well to the range recommended by the MEP [16] increased $10 \%$ and the rest of results approached this range considerably.

Concerning to the runtime simulation, the last refinement on the macro grid by rates between $20 \%$ and $60 \%$ did not result in a significant change in the runtime simulation, being the larger variation approximately 1.5 hour in relation to the refined grid by $20 \%$.

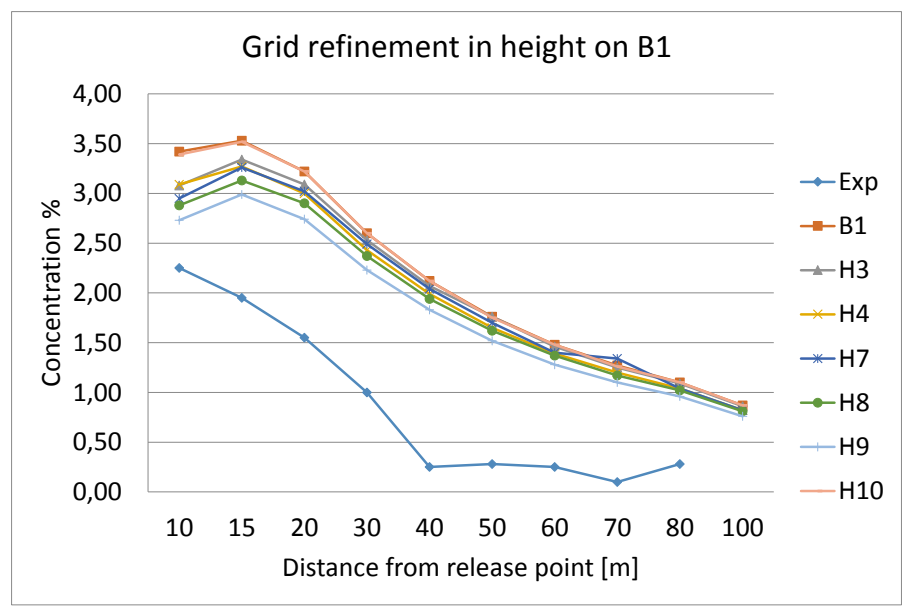

Figure 8 - Comparison among variations in the cells height of the macro grid on B1

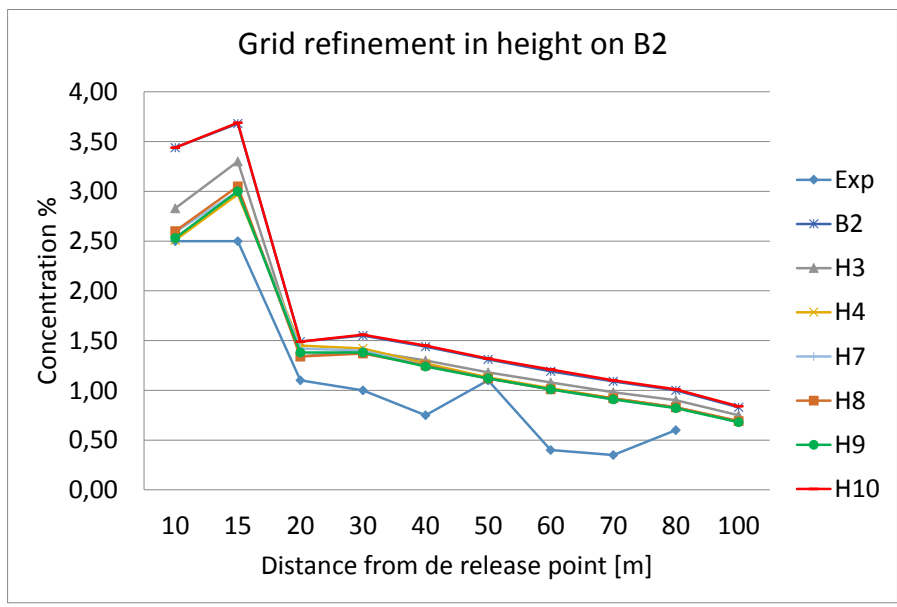

Figure 9 - Comparison among variations in the cells height of the macro grid on B2

\section{CONCLUSIONS AND FURTHER WORK}

This study evaluated, the cloud dispersion from a field test performed by HSL laboratories using a CFD tool and, in particular, performing a dependence grid analysis.

Two trials of the field tests were analyzed; one presenting an unobstructed scenario and the other with a barrier blocking the spread of the cloud. In both scenarios the refinement of the grid improved the results, especially in the region near the discharge and before the obstacle. The variations in the length and width of the cells produced minor effects; then our recommendation is to maintain these dimensions reasonably coarse in order to save runtime simulation. However, height variation of the macro grid cells produced significant effects 
since the refinement in this dimension allows a better representation of the parcel of the weight in the momentum governing equation, which in the case of a dense gas, has a great influence on dispersion.

A dependence grid analysis of the micro grid was next performed. It showed that variations lower than or equal to $\pm 20 \%$ in the micro grid dimensions do not produce significant changes in the results, thus the grid near the source could be fixed at the most at $20 \%$ greater than the recommended by the guidelines in order to save runtime simulation.

Finally, effects of variations by more than $20 \%$ in the macro grid were examined; the refinement in the grid improved significantly the results, at least $12 \%$ comparing the results of the original grid with the grid refined in $50 \%$. However, the aspect ratio among the cells dimensions should be maintained lower than two; if a finer grid is needed, one should consider refining the grid in other directions also. For dispersions analysis involving dense gas, the grid should be stretched in far field in order to reduce the simulation time.It is important to choose a suitable grid especially concerning the height of the cell. For scenarios similar to those discussed here, it is recommended cell heights no greater than $0.5 \mathrm{~m}$ in the region between the release point and the ground.

Future research will imply performing a sensitivity analysis of key parameters on dispersion analysis using CFD tools in order to get even more refined simulations in the analyses of consequences in environments with complex geometry. This will allow a better founded decision making process when setting computational parameters in CFD simulations.

Additionally, further work may explore dependence grid analysis considering unstructured grids and hybrid meshes.

\section{ACKNOWLEDGMENTS}

This paper reports part of the overall results obtained in the R\&D project number 01.10.0498.00 sponsored by FINEP Studies and Projects Financing Agency, a public institution linked to the Ministry of Science and Technology in Brazil, whose support the authors gratefully wish to acknowledge. Author sponsored by CNPq.

The authors gratefully wish to acknowledge the Program for Development of Human Resources (PRH) from Petrobras and Brazilian National Petroleum, Natural Gas and Biofuels Agency (ANP) by the financial support.

\section{REFERENCES}

[1] Cormier, B. R. 2008, “Application of computational fluid dynamics for LNG vapor dispersion". Journal of Loss Prevention in the Process Industries. pp. 332-352.

[2] Middha, P. and Hansen, O. R. 2009, "Using computational fluid dynamics as a tool for hydrogen safety studies". Journal of Loss Prevention in the Process Industries. Vol. 22, pp. 295-302.

[3] Dharmavaram, S., Hanna, S. R. and Hansen, O. R 2005. "Consequence Analysis-Using a CFD model for industrial Sites”. Process Safety Progress. Vol. 24, pp. 316-327.
[4] Mazzoldi, A., Hill, T. and Colls, J. J., 2011,“Assessing the risk for $\mathrm{CO}_{2}$ transportation within $\mathrm{CCS}$ projects, CFD modelling". International Journal of Greenhouse Gas Control, pp. 816-825.

[5] Anderson, J. D. Jr. 1995, “Computational Fluid Dynamics - The basics with applications". Maryland: McGrawHill. pp. 547

[6] Plasmans, J., Donnat, L., Carvalho, E., Debelle, T., Marechal, B., Baillou, F., 2012, "Challenges with the use of CFD for major accident dispersion modeling". Process Safety Progress. Vol.00, pp. 1-5. doi: 10.1002/prs.11571.

[7] FLACS Software - version 9.1. 2013, GexCon AS.

[8] Gavelli, F., Scott, D. G. and Hansen, O. R. 2011, "Evaluating the potential for overpressures from the ignition of a LNG vapor cloud during offloading". Journal of Loss Prevention in the process industries. Vol. 24, pp. 908-915. DOI: 10.1016/j.jlp.2011.07.002.

[9] Launder, B. E. and Spalding E. 1974, "The numerical computation of turbulent flows”. D. B. Vol. 3, pp. 269-289.

[10] HSE - Health and Safety Executive. 2013,"Review of FLACS version 9.0 - Dispersion modelling capabilities". s.1. : Available at: http://www.hse.gov.uk/research/rrpdf/rr779.pdf, accessed on January, 2013.

[11] Thompson, J. F., Soni, . B. K. \& Weatherill, N. P., 2010. Handbook of Grid Generation. Ilustraded ed. s.1.:CRC Press.

[12] Yasushi, I., 2012. "Challenges in unstructured mesh generation for practical and efficient computational fluid dynamics simulations". Computer \& Fluids, pp. 1-6.

[13] Luo, H. \& Spiegel, S., 2010. "Hybrid Grid Generation Method for Complex Geometries". American Institute of Aeronautics and Astronautics Journal, 48(11), pp. 1-9.

[14] Arntzen, B. J., 1998. "Modelling of turbulence and combustion for simulation of gas explosions in complex geometries". Bergen: Thesis for the Doctor engineering degree - The Norwegian university of science and technology.

[15] Butler, C. J. \& Royle, M., 2001. "Experimental data acquistion for validation of a new vapour cloud fire (VCF) modelling approach".pp.1-102: Health and Safety Laboratory HSE.

[16] Coldrick, S., Lea, C. J., \& Ivings, M. J., 2009. "Validation database for evaluating vapor dispersion model for safety analysis of LNG facilities - Review". The fire protection research foundation. Health and Safety Laboratory - HSE. 